\title{
Using a grating structure for phase compensation in achieving an efficient round-trip optical parametric process in periodically poled lithium niobate with an incomplete quasi-phase-matching period
}

\author{
Chih-Wei Hsu and C. C. Yang \\ Institute of Electro-Optical Engineering, National Taiwan University, 1 Roosevelt Road, Section 4, Taipei, Taiwan
}

Received December 3, 1998

\begin{abstract}
An incomplete period of the last quasi-phase-matching (QPM) segment in a periodically poled nonlinear crystal represents a key problem in implementing a nonlinear wavelength-conversion device with round-trip or oscillating waves of interaction. Such a segment at the crystal end implies broken QPM periodicity for the reflected waves of interaction. We demonstrate numerically that with a grating structure at the crystal end for the reflecting signal the wavelength-dependent phase shift at Bragg reflection can compensate for the phase mismatch of the reflected signal, idler, and pump of an optical parametric process. Therefore, by slightly shifting the signal wavelength we can maintain the phase-matching condition with any length of the last QPM segment. It is shown that with a grating structure the conversion efficiency of the round-trip optical parametric process can always be optimized. (c) 1999 Optical Society of America
\end{abstract}

OCIS codes: $190.4410,190.0190,190.4970$.

With the development of domain-inversion techniques for quasi-phase-matched $\mathrm{LiNbO}_{3}$ and other nonlinear optical materials, wavelength-conversion efficiencies for frequency doubling and optical parametric processes have been tremendously increased. ${ }^{1,2}$ With this progress, wavelength conversion with $\mathrm{cw}$ operation has become possible., Meanwhile, although quasi-phase matching (QPM) limits the gain bandwidth of the signal, femtosecond pulses have been implemented in optical parametric oscillators (OPO's) with periodically poled $\mathrm{LiNbO}_{3}$ (PPLN) ${ }^{5,6}$ However, so far all oscillators based on quasi-phase-matched materials for wavelength conversion that we know of have been made with an external-cavity structure. For convenience of application, it is a natural trend to develop a compact monolithic wavelength converter, particularly for cw operation. The first thought in implementing such a device is to prepare a high-reflection coating on the end facet of a quasi-phase-matched material for reduction of the oscillation threshold. However, such an arrangement faces a basic problem, i.e., the length of the last QPM segment at the material end facet is uncontrollable because the period of QPM ranges from a few micrometers to a few tens of micrometers. Unless the length of the last QPM period is exactly equal to one-quarter of the QPM period (the optimized situation), the reflected signal from the end facet will experience broken QPM periodicity and suffer from reduced wavelength-conversion efficiency or even reverse the energy flow of wavelength conversion. One can possibly solve this problem by preparing an antireflection coating for one of the pump, signal, and idler waves. However, since one needs to prepare a high-reflection coating for either one or both of the other waves at the same time to reduce the oscillation threshold, the coating process becomes quite difficult. For second-harmonic generation, slant end facets have been used to solve this problem by transverse translation of the quasi-phase-matched crystal for optimization of the length of the last QPM period. ${ }^{7}$ This method can improve the conversion efficiency in a system with only a round-trip nonlinear interaction; however, it cannot be used to form a compact oscillator because of the slant end facet.

In this Letter we propose the idea of using a grating structure to solve the problem of the incomplete QPM period and demonstrate simulation results of the use of phase compensation in achieving an efficient roundtrip optical parametric process in PPLN. Although chemical wet etching is not a good technique for fabricating corrugation gratings on periodically poled nonlinear crystals because the etching rates of the opposite domains are different, surface gratings on $\mathrm{LiNbO}_{3}$ have been successfully fabricated, with laser ablation. ${ }^{8,9}$ It is suspected that the laser ablation process is weakly dependent on domain inversion.

To obtain numerical results we solved the following coupled equations for the complex amplitudes of the forward and the backward pump, $A_{p}{ }^{+}$and $A_{p}{ }^{-}$, the forward and the backward signal, $A_{s}{ }^{+}$and $A_{s}{ }^{-}$, and the forward and the backward idler, $A_{i}{ }^{+}$and ${A_{i}}^{-}$:

$$
\begin{aligned}
& \frac{\partial A_{s}{ }^{+}}{\partial z}=-j \kappa_{\mathrm{nl}} A_{i}{ }^{{ }^{*}} A_{p}{ }^{+} \exp (-j \Delta k z) \\
& { }^{-} j \kappa A_{s}{ }^{-} \exp (2 j \delta z), \\
& \frac{\partial A_{i}{ }^{+}}{\partial z}=-j \kappa_{\mathrm{nl}} A_{s}{ }^{*}{ }^{*} A_{p}{ }^{+} \exp (-j \Delta k z), \\
& \frac{\partial A_{p}{ }^{+}}{\partial z}=-j \kappa_{\mathrm{nl}} A_{s}{ }^{+} A_{i}{ }^{+} \exp (j \Delta k z), \\
& \frac{\partial A_{s}{ }^{-}}{\partial z}=j \kappa_{\mathrm{n} 1} A_{i}{ }^{-*} A_{p}{ }^{-} \exp (j \Delta k z) \\
& +j \kappa A_{s}{ }^{-} \exp (-2 j \delta z),
\end{aligned}
$$




$$
\begin{gathered}
\frac{\partial A_{i}{ }^{-}}{\partial z}=j \kappa_{\mathrm{nl}} A_{s}{ }^{-*} A_{p}{ }^{-} \exp (j \Delta k z), \\
\frac{\partial A_{p}{ }^{-}}{\partial z}=j \kappa_{\mathrm{nl}} A_{s}{ }^{-} A_{i}{ }^{-} \exp (-j \Delta k z) .
\end{gathered}
$$

Here $\kappa, \kappa_{\mathrm{nl}}, k_{s}, k_{i}$, and $k_{p}$ are the grating coupling coefficient, the nonlinear coupling coefficient, and propagation constants of the signal, idler, and pump, respectively. Also, the nonlinear phase mismatch and grating phase mismatch are described by $\Delta k=$ $k_{p}-k_{s}-k_{i}$ and $\delta=k_{s}-\pi / \Lambda$, respectively, with $\Lambda$ representing the grating period. Note that only the signal waves, $A_{s}{ }^{+}$and $A_{s}{ }^{-}$, experience Bragg reflection of the grating, as described by Eqs. (1) and (4). The possible effects of the grating on the pump and the idler waves are ignored.

To demonstrate the effects of a grating structure we compare the results obtained with the two devices, the layouts of which are shown in Fig. 1. Figures 1(a) and 1(b) show two PPLN crystals with formed waveguides. In both cases there is a QPM segment of incomplete period with length $L_{i}$ on the right-hand side. In the case shown in Fig. 1(a), a coating is prepared for high reflection of the signal wave. In the case shown in Fig. 1(b), a surface grating with a length $L_{g}$ is fabricated. The pump wave is incident from the left-hand side. We are also concerned with the signal output from the left-hand side. For our numerical study we consider the pump wavelength $1064 \mathrm{~nm}$ and the signal wavelength $1550 \mathrm{~nm}$. The idler wavelength is thus $3.393 \mu \mathrm{m}$. The corresponding QPM period of PPLN is $\Gamma=29.6 \mu \mathrm{m}$. To achieve a more-than- $2 \pi$ phase variation over the Bragg reflection window we considered a linearly chirped grating with the central grating period at $\Lambda=361.83 \mathrm{~nm}$, which corresponds to the Bragg wavelength $1550 \mathrm{~nm}$. The grating has a length of $0.8 \mathrm{~mm}$, a chirp of $4.7375 \mathrm{~nm} / \mathrm{mm}$, and a coupling coefficient of $4000 \mathrm{~m}^{-1}$. This grating provides 95\% reflectance at $1550 \mathrm{~nm}$ in linear optics. For reasonable comparison, the reflectance of the signal from the coating in Fig. 1(a) is set to be close to that from the grating when the nonlinear effect is included. The PPLN length is $\sim 2 \mathrm{~cm}$ and is slightly adjusted for different cases so that we can consider various sizes of $L_{i}$. Generally, the PPLN length of the device with the coating is nearly equal to the length of the plain PPLN plus the penetration length into the grating of the device with the grating. In conducting the simulations we assumed that there exists a waveguide for the pump, the signal, and the idler. Guiding for all three waves is an issue deserving further investigation. Here, for mathematical simplification and without loss of generality, we assume that the poor overlap of the modal profiles of these three waves leads to an efficiency of nonlinear conversion that is lower the case of ideal overlap by a factor of $10 \%$.

Figure 2 shows the reflectance and the phase shift of the chirped grating as a function of wavelength. It describes the case of the device in Fig. 1(b) with $L_{i}=0$, an input pump intensity of $50 \mathrm{MW} / \mathrm{cm}^{2}$, the pump reflectance of the right-hand end facet at unity, and the signal and the idler reflected by the uncoated right-hand end facet. Also, the initial signal and idler intensities on the left-hand side are set at 0 and $500 \mathrm{~W} / \mathrm{cm}^{2}$, respectively. The grating chirp is designed so that the phase shift over the Bragg reflection window is larger than $2 \pi$. One can see that the nonlinear effect narrows the reflection window and enhances the reflectance in the narrowed window.

Figure 3 shows the output signal intensity as a function of the signal wavelength within the Bragg reflection window for various $L_{i}$ values. On the right-hand side of the grating device, the signal experiences uncoated end-facet reflection besides the Bragg reflection from the grating. Meanwhile, the idler and the residual pump are reflected from the uncoated end facet. The pump intensity is fixed at $50 \mathrm{MW} / \mathrm{cm}^{2}$. The initial signal and idler intensities on the left-hand side are set at 0 and $500 \mathrm{~W} / \mathrm{cm}^{2}$, respectively. One can see that with the coating the output signal intensity varies significantly with $L_{i}$. From the optimum case of $L_{i}=0.25 \Gamma$ to the worst case of $L_{i}=0$, there is a more-than-75\% difference. On the other hand, with the grating the output signal intensity oscillates with wavelength. The maximum of the oscillation reaches the level of the optimum situation of the coating device, and the minimum has a level that is roughly the same as the worst situation of the coating device. The oscillating output signal intensity is attributed to the signal phase variation with wavelength when the signal is reflected from the grating. At the peaks, the phase

(a) PPLN

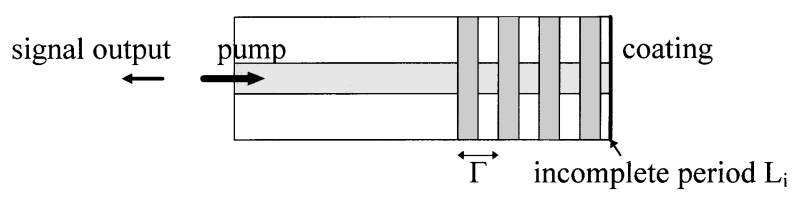

(b)

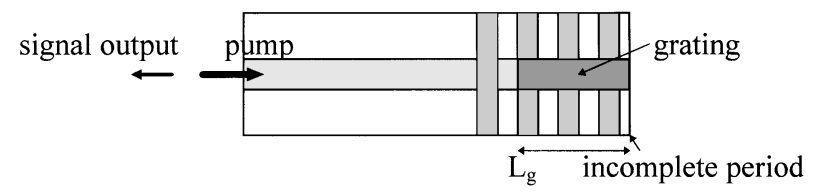

Fig. 1. Layouts of the two devices for comparison: (a) device with the coating, (b) device with a grating structure.

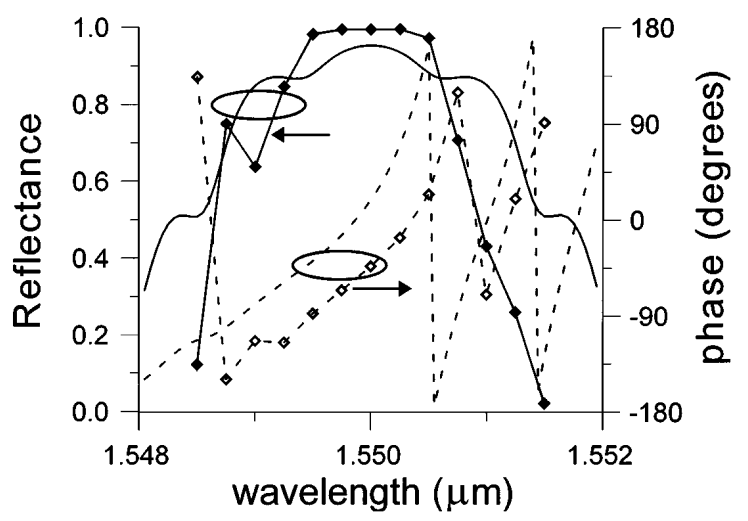

Fig. 2. Reflectance and phase shift of the reflected wave from the chirped grating without (curves without symbols) and with (curves with symbols) the nonlinear effect. 


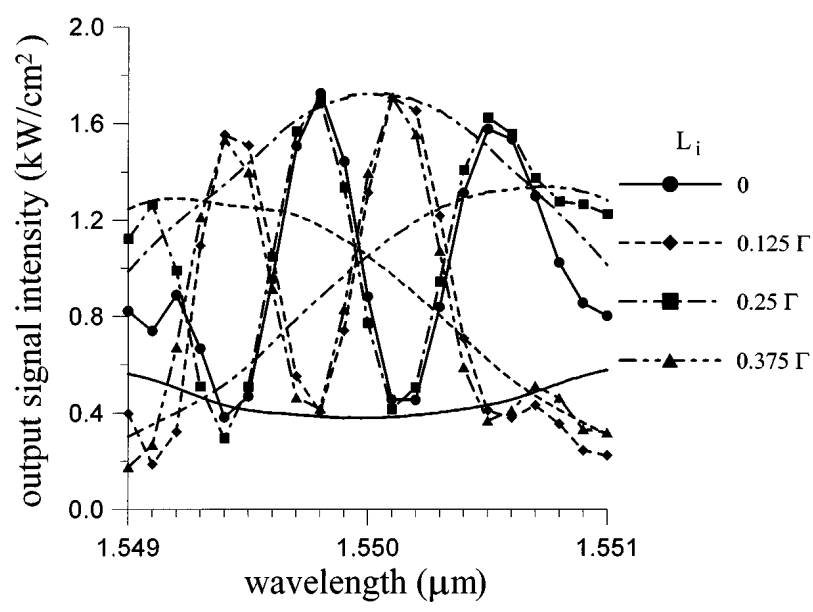

Fig. 3. Output signal intensity as a function of wavelength for four different $L_{i}$ values. The curves without symbols and with symbols represent the results for the coating and the grating devices, respectively.

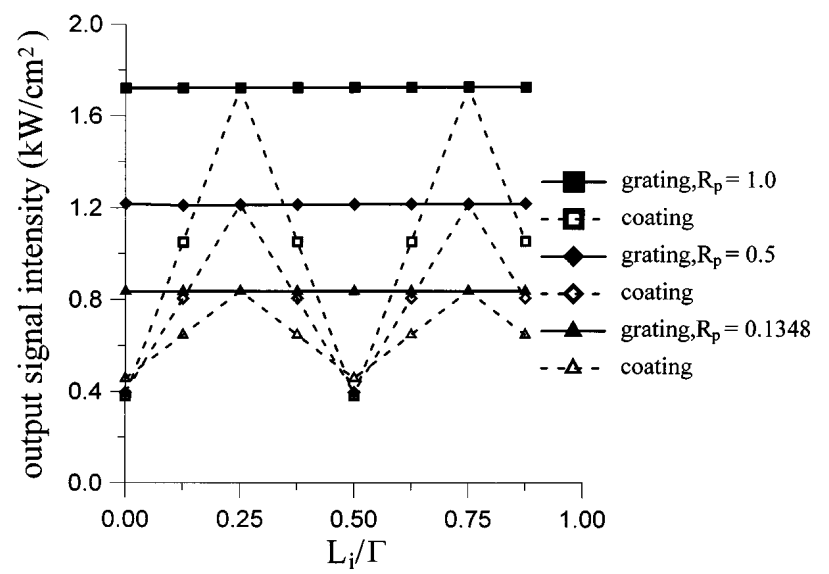

Fig. 4. Output signal intensity as a function of $L_{i}$ for three different pump-wave reflectance levels $R_{p}$ at the righthand facet. The solid and open symbols represent the results for the grating and the coating devices, respectively.

of the reflected signal has a value such that the phasematching condition among the reflected pump, signal, and idler is reached for the optimum nonlinear conversion. The results in Fig. 3 imply that with the grating structure the system will provide the output with the optimum nonlinear conversion by slightly shifting the signal wavelength within the Bragg reflection window of the grating. The wavelength shift is quite small, as small as a few tenths of a nanometer. Such a shift is always within the QPM window for efficient nonlinear conversion.

In Fig. 4, we show the output signal intensity as a function of $L_{i}$. The symbols represent the three different end-facet pump-wave reflectance levels. $R_{p}=$ 0.1348 corresponds to uncoated end-facet reflection. For comparison, the three pump-wave reflection levels apply to both the grating and the coating devices. The data points for the grating device correspond to the results of the highest peaks in Fig. 3. In Fig. 4, one can see the oscillating feature with $L_{i}$ in the case of the coating device. However, in the grating device the output signal intensity is always maintained at the optimum level. Because the oscillating amplitude of the coating device increases with pump reflectance, the improvement of nonlinear conversion becomes more significant with a larger pump reflectance.

In summary, we have numerically demonstrated that when we use a grating structure on one end of a PPLN crystal the wavelength-dependent phase shift at the Bragg reflection can compensate for the phase mismatch among the backward-propagating signal, idler, and pump waves because of an incomplete period of the last QPM segment. By slightly shifting the signal wavelength, we can achieve phase compensation, and the nonlinear wavelength conversion in the backward direction can be enhanced. Such a phase-compensation mechanism not only can increase the overall conversion efficiency in a round-trip optical parametric process but also can be used to implement a monolithic OPO. Note that in implementing a monolithic OPO with gratings at the two ends of the resonator the differences between the two gratings and between the last two QPM segments lead to two different optimum wavelengths. Therefore, one needs to compromise between the two different optimum wavelengths in such an OPO to reach the optimum oscillating wavelength. This is one of our current research topics.

This research was sponsored by National Science Council, Republic of China, grants NSC 882215-E-002-012, NSC 87-2215-E-002-006, and NSC 87-2215-E-002-007. C. C. Yang's email address is ccy@cc.ee.ntu.edu.tw.

\section{References}

1. G. D. Miller, R. G. Batchko, W. M. Tulloch, D. R. Weise, M. M. Fejer, and R. L. Byer, Opt. Lett. 22, 1834 (1997).

2. L. E. Myers and W. R. Bosenberg, IEEE J. Quantum Electron. 33, 1663 (1997).

3. T. J. Edwards, G. A. Turnbull, M. H. Dunn, M. Ebrahimzadeh, H. Karlsson, G. Arvidsson, and F. Laurell, Opt. Lett. 23, 837 (1998).

4. M. E. Klein, D.-H. Lee, J.-P. Meyn, B. Beier, K.-J. Boller, and R. Wallenstein, Opt. Lett. 23, 831 (1998).

5. K. C. Burr, C. L. Tang, M. A. Arbore, and M. M. Fejer, Appl. Phys. Lett. 70, 3341 (1997).

6. C. McGowan, D. T. Reid, Z. E. Penman, M. Ebrahimzadeh, and W. Sibbett, J. Opt. Soc. Am. B 15, 694 (1998).

7. G. Imeshev, M. Proctor, and M. M. Fejer, Opt. Lett. 23, 165 (1998).

8. C. Y. Chao, C. Y. Chen, C. W. Liu, Y. Chang, and C. C. Yang, Appl. Phys. Lett. 71, 2442 (1997).

9. G. P. Luo, Y. L. Lu, Y. Q. Lu, X. L. Guo, S. B. Xiong, C. Z. Ge, Y. Y. Zhu, Z. G. Liu, N. B. Ming, J. W. Wu, D. S. Ding, and Z. H. Lu, Appl. Phys. Lett. 69, 1352 (1996). 